may reverse both the EEG and cognitive, language, and behavioral regression. The well known tendency to development of tolerance with use of benzodiazepines was considered unusual in the treatment of ESES in this study. Seizures and the ESES may be exacerbated by carbamazepine.

\title{
VALPROATE-INDUCED WEIGHT GAIN
}

To determine the relation of carnitine and insulin levels to weight gain during valproate therapy of epilepsy in children, twenty patients were randomly assigned to receive either carnitine or placebo supplementation, in a study at Hacettepe University, Ankara, Turkey. The mean age was 8.3 and 9.4 years in the two groups. Seizures were completely controlled. Weight gain occurred in both groups after a 3 month period of observation. Weight gain did not correlate with carnitine levels. Mean insulin levels and insulin/glucose ratios were increased during valproate therapy. Weight gain might be related to the decrease in glucose levels and consequent appetite stimulation. (Demir E, Aysun S. Weight gain associated with valproate in childhood. Pediatr Neurol May 2000;22:361-364). (Respond: Dr Sabiha Aysun, Department of Pediatric Neurology, Hacettepe University, Ankara 06100, Turkey).

COMMENT. Weight gain associated with valproate therapy for epilepsy is not related to carnitine deficiency. It may be caused by appetite stimulation, secondary to higher insulin and lower blood glucose levels. VPA may decrease glucose levels by inhibiting gluconeogenesis, and by other possible mechanisms.

\section{TOXIC-METABOLIC DISORDERS}

\section{PRENATAL ALCOHOL AND NEUROLOGIC DYSFUNCTION}

Of 698 pregnant women interviewed about alcohol consumption at the maternity hospital in Roubaix, France, 156 of the offspring were investigated at age 4 and $1 / 2$ years, using a standardized neuropsychological assessment. A posture score was not related to alcohol consumption, whereas a lower general cognitive index (GCI) and a minor neurological sign score were directly related to

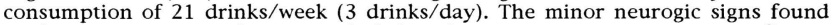
at the neuromotor evaluation included synkinesis or mirror movements, fingernose incoordination, hopping dyspraxia, and impaired ability to walk on heels. The high number of neurologic abnormalities was associated with prenatal alcohol consumption, after controlling for a lower GCI. (Larroque B, Kaminski M, Dehaene P, Subtil D, Querleu D. Prenatal alcohol exposure and signs of minor neurological dysfunction at preschool age. Dev Med Child Neurol August 2000;42:508-514). (Respond: Beatrice Larroque MD PhD, Institut National de la Sante et de la Recherche Medical Unit 149, 16 Avenue Paul Vaillant, Couturier, 94807 Villejuif Cedex, France).

COMMENT. Children born to mothers who consumed 21 or more alcoholic drinks per week ( $11 \%$ of the sample) had more minor neurologic abnormalities than those exposed to less alcohol. Levels of alcohol consumption lower than those associated with fetal alcohol syndrome can cause impairments of neurologic function recognized on clinical examination.

Twelve-year follow-up of children exposed to alcohol in utero, at the University of Helsinki, found that the longer the exposure and the more severe the fetal alcohol syndrome, the more often the children required special 